\title{
Hidatidosis humana: Generalidades y situación epidemiológica en Chile según egresos hospitalarios y notificación obligatoria entre los años 2001 y 2005
}

\author{
Sandra Cortés A. y Carla Valle B.
}

\section{Human hydatidosis: General aspects and epidemiological situation in Chile according to hospital discharge and mandatory reporting from 2001 to 2005}

Human hydatidosis in Chile is described using as a source of information the Disease Notification Systems and hospital discharges between 2001 and 2005. To assess the extent of human infection we calculated incidence rates and hospital discharges during this period by geographical region. Incidence rate for the period was 2.2 per 100,000 inhabitants with higher rates in the regions of Coquimbo, La Araucanía and Magallanes. The hospital discharge rate for the period was 6 per 100.000 inhabitants, being the most affected regions: La Araucanía, Aysén and Magallanes. 58.8\% of cases reported are from Echinococcus granulosus, while $43.6 \%$ were registered as unspecified echinococcosis. The notification system provides a real estimate of the magnitude of this disease, reporting cases with diagnostic confirmation. The rates obtained from the discharge system overestimate the magnitude of this disease. The improvement of the notification system and seroprevalence studies are recommended.

Key words: Hydatidosis, echinococcosis, rates, notification, hospital discharges.

Palabras clave: Hidatidosis, equinococosis, tasas, notificación, egresos hospitalarios.

\section{Introducción}

L a equinococosis quística o hidatidosis es una zoonosis de distribución mundial producida por céstodos del género Echinococcus ${ }^{1}$ considerada una enfermedad de gran importancia en salud pública por las elevadas pérdidas que produce en los sistemas de salud asociados a dificultades diagnósticas y a los altos costos de tratamiento de las personas. Además se destaca su impacto económico debido a las pérdidas por decomiso de vísceras animales afectadas ${ }^{2}$. El presente trabajo se organiza en dos partes; en la primera se presentan las características generales, diagnósticos, tratamientos y consideraciones epidemiológicas de esta enfermedad, mientras que en la segunda parte se describen los principales indicadores de esta enfermedad en Chile según dos fuentes de información, esto es, bases de egresos hospitalarios y sistema de notificación obligatoria de enfermedades, ambas del período 2001 al 2005, destacando sus fortalezas y debilidades y su rol en la toma de decisiones en salud pública.

\section{Características generales de la hidatidosis}

Se reconocen cuatro especies patógenas para el hombre: E. granulosus, E. multilocularis, E. oligarthus y E. vogeli ${ }^{1}$. La especie multilocularis, causante de la hidatidosis alveolar, prevalece en zonas árticas de América del Norte y se transmite a través de lobos y zorros ${ }^{3}$. Echinococcus vogeli y E. oligarthus causan hidatidosis poliquística, y se encuentra en Centroamérica y norte de América del Sur, manteniéndose por ciclos con hospederos silvestres ${ }^{4}$. Echinococcus granulosus causa la equinococosis quística y es la única especie identificada en Chile ${ }^{5}$. Aun cuando han sido reportados dos casos (uno en 1984 y otro en 2004) causados por E. multilocularis ${ }^{3}$, para Serra $^{6}$ la hidatidosis alveolar es una enfermedad extraña a Chile, siendo posible que entidades poliquísticas causadas por E. vogeli y E. oligarthus sean confundidas con hidatidosis alveolar ${ }^{6}$.

El parásito requiere de un hospedero definitivo (carnívoro, especialmente el perro) en cuyo intestino se desarrolla la fase adulta o estrobilar y un hospedero intermediario (ovino, caprino, bovino o porcino) para el desarrollo de las larvas o metacéstode, en forma de quiste ("quiste hidatídico") especialmente en hígado y pulmón ${ }^{2}$. El humano actúa como hospedero intermediario accidental $^{1}$.

El hombre se infecta al ingerir huevos fértiles adheridos al ano o pelos de perros parasitados o por la ingestión de verduras o aguas contaminadas con materia fecal canina ${ }^{2}$.

El crecimiento del quiste hidatídico depende del potencial evolutivo del embrión hexacanto, del tejido
Pontificia Universidad Católica de Chile.

Departamento de Salud Pública (SCA).

Universidad de Viña del Mar, Chile.

Facultad de Ciencias

Agropecuarias (CVB)

Financiamiento: El estudio se realizó sin aportes económicos o becas.

Basado en la tesis: “Descripción de la notificación obligatoria de hidatidosis humana y su relación con los egresos hospitalarios en Chile durante el período 2001 al 2005", aprobada en Agosto de 2008.

Facultad de Ciencias Agropecuarias. Universidad de Viña del Mar, Chile para obtener el grado de licenciado en Medicina Veterinaria de Carla Valle Barra, titulada.

Recibido: 7 de Julio 2009 Aceptado: 8 de mayo 2010

Correspondencia a: Sandra Cortes A. scortes@med.puc.cl 
circundante y de la resistencia del hospedero. Puede ser muy rápido (5 ó $10 \mathrm{~cm}$ en pocos años) y generar síntomas graves con riesgo de muerte para el portador o puede comportarse en forma benigna, crecer no más de 2 a $7 \mathrm{~cm}$ y envejecer con su portador sin producir daño a la salud $d^{7,8}$.

Las localizaciones anatómicas de los quistes pueden dar lugar a diversas patologías de muy variable intensidad y gravedad. Algunas pueden ser asintomáticas, cuando no interfieren con el normal funcionamiento de los órganos donde se implantan. Dentro de las localizaciones, la más frecuente es la hepática, seguida por la pulmonar. Sin embargo, su ubicación está determinada por las defensas del hospedero, las que varían según especie, edad e individualidad 9 .

Los síntomas varían de acuerdo al órgano afectado y a la presencia de complicaciones. Los órganos más afectados son el hígado (50-70\% de los casos), habitualmente en el lóbulo hepático derecho $(80 \%$ lesión única y $20 \%$ lesiones múltiples); pulmón 20-40\% (60\% pulmón derecho y $13 \%$ es bilateral) y otras localizaciones (10\%). Ningún órgano es inmune a la infección, describiéndose lesiones peritoneales, esplénicas, renales, óseas, tiroideas y mamarias ${ }^{10,11}$.

Los síntomas más frecuentes producidos por el quiste hepático incluyen dolor en el hipocondrio derecho, masa palpable, ictericia y fiebre. Las complicaciones más frecuentes de los quistes hepáticos suelen ser las roturas e infección, transformándose en un absceso ${ }^{1}$. En la localización pulmonar dolores de pecho, fatiga, cansancio $\mathrm{y}$ tos $^{2}$. Los quistes pulmonares suelen presentar vómica asociada a hemoptisis, ya que hasta $50 \%$ de las lesiones pulmonares se encuentran complicadas al momento del diagnóstico. La secreción bronquial acompañada de bilis es un signo muy poco frecuente pero patognomónico de los quistes hepáticos que migran al tórax y causan una fístula bilio-bronquial. El shock anafiláctico y la siembra peritoneal o pleural suelen observarse en el caso de roturas espontáneas o durante el tratamiento quirúrgico ${ }^{12,13}$.

\section{Diagnóstico y tratamiento}

El diagnóstico de la hidatidosis humana se fundamenta en la información clínica y epidemiológica, y en el uso de métodos imagenológicos tales como la radiología, la ultrasonografía, la tomografía computarizada y la resonancia magnética ${ }^{14}$. En el hemograma el hallazgo más frecuente es la eosinofilia (más de 5\% o más de 300 células $/ \mathrm{mm}^{3}$ ), mientras que un perfil hepático con elevación de las transaminasas y/o hiperbilirrubinemia sugiere complicaciones del quiste o compromiso de la vía biliar (rotura, abscedación) ${ }^{1}$.

Las pruebas serológicas permiten un diagnóstico específico pero, para que tengan valor, se requiere de una reacción antígeno/ anticuerpo, obtenida por la capacidad de respuesta inmunológica del hospedero y el contacto de este sistema inmunocompetente con los antígenos (fisura o rotura de la capa germinativa). Ninguna de las técnicas mencionadas permite por sí sola el diagnóstico certero por lo que suelen asociarse al menos dos de ellas, siendo muy útiles en el seguimiento posterior al tratamiento y en estudios epidemiológicos ${ }^{1,14}$.

En pacientes sintomáticos con hidatidosis confirmada por cirugía se ha reportado una sensibilidad con valores entre $80 \%$ para hemoaglutinación indirecta (HAI), 82 a $88 \%$ para doble difusión cinco (DD5), 88 a 96\% para el ensayo inmunoenzimático (ELISA) y $92 \%$ para electroinmunotransferencia. La especificidad de estos métodos varía desde 95 a $100 \%{ }^{15,16}$.

En portadores humanos sin sintomatología, la posibilidad de detectar una respuesta serológica positiva es menor ante el predominio de quistes pequeños y no complicados, reportándose una sensibilidad con DD5 de $31 \%$ y con ELISA $63 \%$ utilizándose estudios completos por imágenes como prueba de referencia ${ }^{15}$.

La equinococcosis quística ha sido considerada tradicionalmente una enfermedad de resolución quirúrgica que incluye la eliminación de el (los) quiste(s), corrección de los efectos de la presencia del quiste en el órgano afectado (periquística, cavidad residual, etc) y el tratamiento de complicaciones que el quiste ha provocado por su evolución (fístulas biliares, pleurales, siembra peritoneal, pleural, etc) ${ }^{1}$. Sin embargo, también hoy se dispone de tratamientos quirúrgicos mini invasores, tal como la punción (PAIR) ecoasistida con aspiración del contenido del quiste, inyección de sustancia escolicida y reaspiración del líquido y cirugía laparascópica, técnicas que permiten acortar en gran medida los períodos de internación de los pacientes y el tratamiento médico con albendazol (carbamato de bencimidazol) utilizado para evitar recidivas en casos de quistes múltiples y de gran tamaño ${ }^{2,17}$.

\section{Aspectos epidemiológicos}

Esta es una enfermedad de notificación obligatoria diaria en Chile, a partir del año 2000. Se notifica una vez confirmado el diagnóstico por el respectivo establecimiento asistencial o por médicos particulares, enviándose al servicio de salud de la zona el formulario de "Enfermedades de Notificación Obligatoria" el mismo día de la confirmación; luego esta información es remitida al Ministerio de Salud semanalmente ${ }^{18,19}$.

El sistema de registro hospitalario produce información estadística de las causas de egresos hospitalarios y datos generales del paciente durante todo el período de hospitalización, es decir, mientras permanece ocupando una cama de un establecimiento autorizado para este fin $^{20}$.

La OPS hace referencia a la actual sub-notificación existente evidenciada por la disparidad de cifras entre los casos notificados y los egresos hospitalarios por 
hidatidosis ${ }^{14}$. Se reporta según el sistema de notificación una incidencia de hidatidosis humana de 2 a 2,5/100.000 hbtes ${ }^{14}$, mientras que según el sistema de egresos hospitalarios se calcula en 9,7/100.000 hbtes $^{21}$.

Esta disparidad de datos requiere ser confirmada, a fin de concentrar los esfuerzos y los análisis posteriores sólo en fuentes que permitan una óptima caracterización de esta enfermedad para el enfoque de acciones preventivas.

\section{Indicadores de morbilidad de hidatidosis en Chile entre los años 2001 y 2005}

En las siguientes secciones se describen los principales aspectos metodológicos para describir el patrón de ocurrencia de esta enfermedad en Chile durante el período 2001-2005.

\section{Material y Método}

Se utilizaron dos fuentes de información secundarias para los años 2001 al 2005 proporcionadas por el Departamento de Estadísticas e Información de la Salud (DEIS), del Ministerio de Salud de Chile:

- Base de datos de enfermedades de notificación obligatoria (ENO), vigente desde el año 2000.

- Base de datos de los egresos hospitalarios.

Este estudio descriptivo se realizó durante el año 2007, fecha en la que sólo se encontraban validadas las bases del sistema ENO para el período 2000 al 2005, por lo que se determinó analizar el período 2001 al 2005 para ambas bases.

No se consideraron otras fuentes de información, tales como los registros de mortalidad (por representar el peor escenario) o de laboratorio (no disponibles al recolectar las bases), los que sin duda hubieran complementado la información presentada en este trabajo.

Se seleccionaron los sujetos con diagnóstico según la CIE-X(22) correspondiente a E. granulosus (B67.0 a B67.4), E. multilocularis (B67.5 a B67.7) y equinococosis no especificada (B67.8 y B67.9).

Se calcularon las tasas de incidencia y de egresos hospitalarios de equinococosis quística en humanos mediante las fórmulas:

Tasa de incidencia $=$

$\underline{\mathrm{N}^{\circ} \text { de enfermos nuevos X } 100.000 \text { hbtes }}$ Población en riesgo

Tasa de egresos $=$

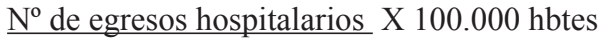
Población en riesgo

Los datos poblacionales correspondieron a las proyecciones de población por grupos de edad y sexo, para todos los servicios de salud y comuna, del período $1990-2005^{23}$.
Se calcularon proporciones de casos notificados y de egresos hospitalarios según grupos de edad, sexo y especie de Equinococcus y la variación temporal y geográfica de las tasas de incidencia y de egresos hospitalarios durante el período en estudio y las regiones de Chile vigentes al realizar el estudio.

Se usaron los programas Microsoft Excel 2002 y SPSS versión 15.0 .

\section{Resultados}

En las Tablas 1 y 2 se muestran las principales características de las personas afectadas y su distribución por grupos de edad y sexo.

La mayoría de los casos notificados se encuentran entre los 30 y 59 años de edad, concentrando $45 \%$ de los casos. De manera similar, según los egresos hospitalarios este grupo etario constituyendo $44 \%$ del total, sin diferencias significativas respecto a los casos notificados. El promedio de edad de los casos notificados es de 38 años, mientras que los egresos tienen en promedio 39 años (Tabla 1). Llama la atención la presentación de casos en edades extremas.

En ambas fuentes el sexo más afectado es el masculino, con 50,9\% según las notificaciones y en 52,4\% según los egresos hospitalarios (Tabla 2).

Tabla 1. Casos notificados y egresos hospitalarios por hidatidosis humana según grupos de edad, Chile, período 2001 al 2005

\begin{tabular}{|lcccc|}
\hline & \multicolumn{2}{c}{ Notificaciones } & Egresos & \\
Edad (años) & $\mathbf{n}$ casos & $\%$ & n casos & $\%$ \\
\hline $0-9$ & 183 & 11 & 397 & 8 \\
\hline $10-19$ & 239 & 14 & 649 & 14 \\
\hline $20-29$ & 199 & 12 & 629 & 13 \\
\hline $30-39$ & 272 & 16 & 726 & 15 \\
\hline $40-49$ & 256 & 15 & 725 & 15 \\
\hline $50-59$ & 232 & 14 & 684 & 14 \\
\hline $60-69$ & 189 & 11 & 504 & 11 \\
\hline $70-79$ & 103 & 6 & 339 & 7 \\
\hline 80 y más & 30 & 2 & 84 & 2 \\
\hline Total & 1.703 & 100 & 4.737 & 100 \\
\hline
\end{tabular}

Tabla 2. Casos notificados y egresos hospitalarios por hidatidosis humana según sexo, Chile, período 2001 al 2005

\begin{tabular}{|lcccc|}
\hline & \multicolumn{2}{c}{ Notificaciones } & \multicolumn{2}{c|}{ Egresos } \\
Sexo & n casos & $\%$ & n casos & $\%$ \\
\hline Mujeres & 838 & 49,1 & 2.255 & 47,6 \\
Hombres & 869 & 50,9 & 2.487 & 52,4 \\
\hline Total & 1.707 & 100 & 4.742 & 100 \\
\hline
\end{tabular}


Tabla 3. Casos notificados y egresos hospitalarios de hidatidosis según especie de Echinococcus, Chile, período 2001-2005

\begin{tabular}{lcccc} 
Especie de Echinococcus & \multicolumn{2}{c}{ Notificaciones } & \multicolumn{2}{c|}{ Egresos } \\
& $\mathbf{n}$ casos & \% & n casos & $\%$ \\
E. granulosus & 935 & 54,8 & 503 & 10,6 \\
E. multilocularis & 27 & 1,6 & 10 & 0,2 \\
No especificada & 745 & 43,6 & 4229 & 89,2 \\
\hline Total & 1.707 & 100,0 & 4.742 & 100,0 \\
\hline
\end{tabular}

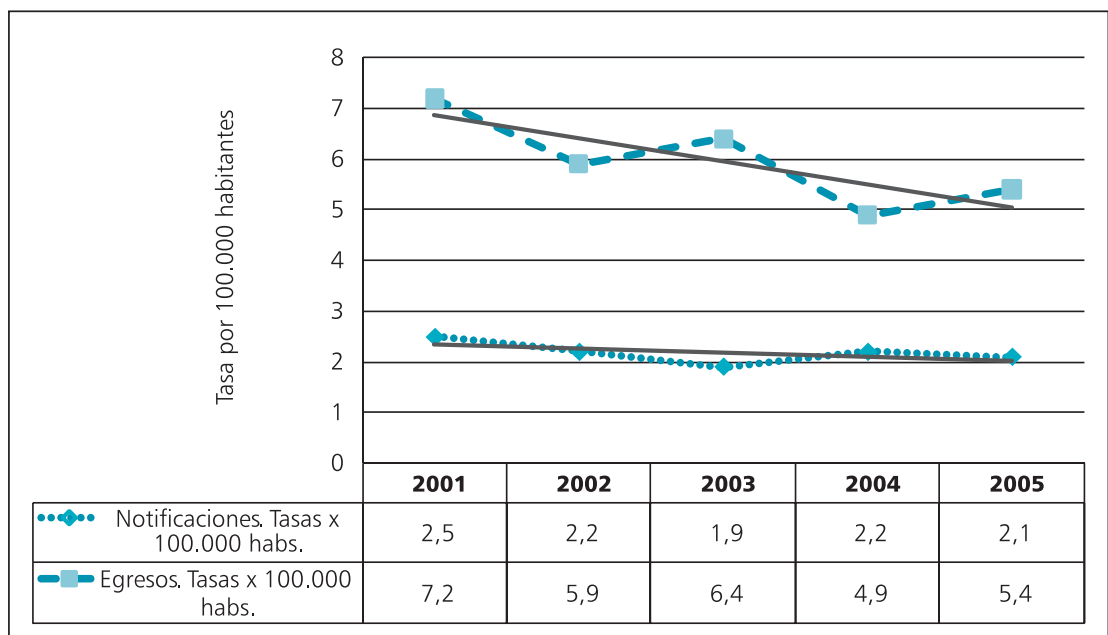

Figura 1. Variación anual de las tasas de incidencia y egresos hospitalarios de hidatidosis humana, Chile, período 2001 al 2005.

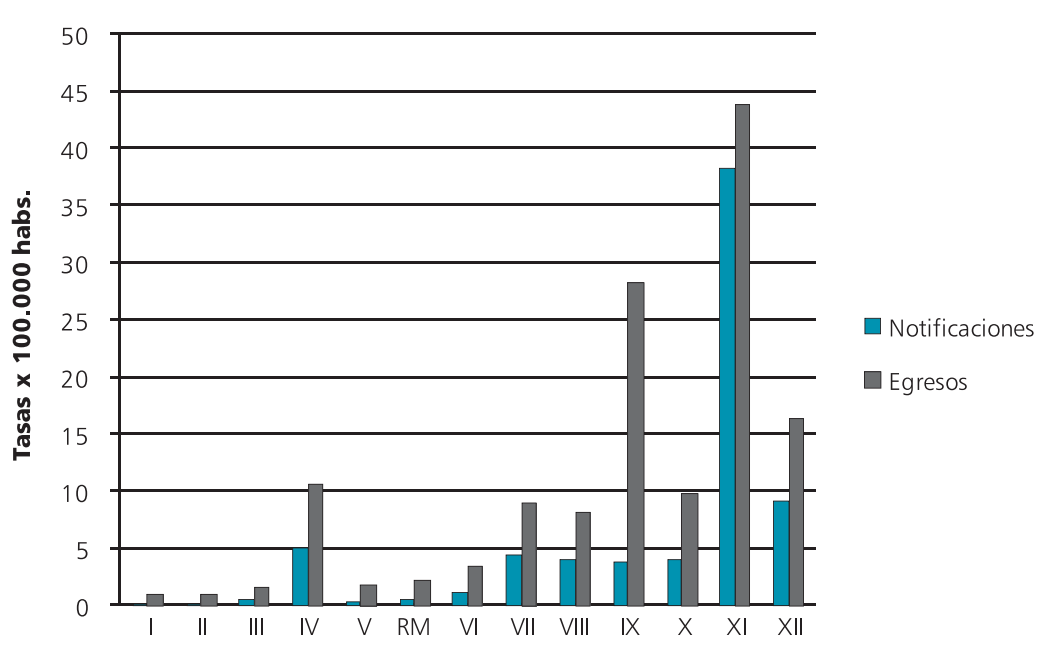

Figura 2. Variación geográfica de las tasas de incidencia y egresos hospitalarios de hidatidosis humana, según regiones de Chile, período 2001- 2005.
La Tabla 3 muestra los agentes etiológicos detectados en ambos sistemas.

El $54,8 \%$ de los casos notificados por hidatidosis son producidos por E. granulosus, mientras que esta especie es detectada sólo en $11 \%$ de los egresos. Se destaca que en $43,6 \%$ de los casos notificados no se especifica el agente causal de hidatidosis, mientras que en los casos egresados asciende a $89,2 \%$ (Tabla 3 ).

La Figura 1 muestra la distribución temporal de las tasas de incidencia y egresos hospitalarios en el período en estudio.

Se registraron 1.707 pacientes confirmados por hidatidosis obtenidos del sistema de notificación obligatoria, con un promedio anual de 341 casos y una tasa promedio de 2,2/100.000 hbtes. Según los egresos hospitalarios se obtuvo un total de 4.742 egresos por equinococosis con un promedio anual de 948 casos y una tasa promedio de 6/100.000 hbtes en el período.

Las tasas anuales de incidencia obtenidas de la notificación obligatoria evidencian una tendencia a la estabilidad en el período, mientras que según el sistema de egresos se observa una tendencia al descenso (Figura 1). En términos generales, las tasas de egresos son más del doble de las tasas de incidencia.

Respecto a la distribución geográfica, según los casos notificados la región más afectada es Bío-Bío (VIII) concentrando $23,1 \%$ de los casos; le siguen las regiones de La Araucanía (IX) con 13,2\% y Maule (VII) con 12,1\%. Las tasas de incidencia más altas se registraron en Aysén (XI), Magallanes (XII) y Coquimbo (IV) con una tasa de 38,$2 ; 9,2$ y $5 / 100.000$ hbtes, respectivamente.

Los egresos hospitalarios indican a Región de La Araucanía como la más afectada, con $27 \%$ de los casos, seguidas por Bío-Bío con $16,7 \%$ y la Región Metropolitana (RM) con $11,7 \%$. Las regiones de Aysén, La Araucanía y Magallanes presentaron la mayor tasa de egresos en el período, con un promedio anual de 43,8 ; 28,1 y 16,4/100.000 hbtes, respectivamente (Figura 2).

\section{Discusión}

Las personas más afectadas por esta enfermedad poseen entre 30 y 49 años de edad, concentrando éstos 30,9 y $30,6 \%$, según los casos notificados y egresos hospitalarios, respectivamente. Estos son individuos en plena edad productiva y por tanto con potencial impacto en su actividad laboral, debido a ausentismo y licencias médicas. Sólo como un antecedente más, Lorca y $\operatorname{cols}^{24}$, estimaron que en pacientes de 23 a 61 años con hidatidosis hepática los costos asociados sólo por diagnóstico y tratamiento fueron equivalentes a US $\$ 1.600$ (valor mediano).

De acuerdo a lo observado, podría ser de interés revisar con más profundidad la ocurrencia de aquellos 
casos reportados en edades extremas, y sus posibles consecuencias, diferentes al grupo en edad productiva.

A pesar de que sólo se describe en Chile E. granulosus ${ }^{5}$ diagnosticada en $54,8 \%$ de los casos notificados, existe $1,6 \%$ de los casos causados por E. multilocularis. Esto podría deberse como señala Serra ${ }^{6}$, a que éstos casos pueden estar confundidos con equinococosis poliquística, entidad causada por E. vogeli y E. oligarthus, especies que han sido descritas en Centroamérica y Norteamérica y que, según el autor, también afectan a países del Cono sur $^{6}$, al contrario de E. multilocularis responsable de la hidatidosis alveolar, descrita solamente en zonas árticas de América del Norte $^{3}$. Llama la atención que existe 43,6\% de los casos notificados diagnosticados como equinococosis no especificada, lo que evidencia la necesidad de capacitación, actualización e investigación en torno al tema por parte del equipo de salud responsable del diagnóstico y su confirmación.

El análisis descriptivo de las bases de datos utilizadas en este estudio muestra que el número de casos reportados en los egresos hospitalarios difiere notoriamente de los obtenidos a partir del sistema de notificación obligatoria. Sin embargo, esta situación no demuestra que efectivamente exista una subnotificación considerando que los casos incorporados a la base de "Enfermedades de Notificación Obligatoria" son casos con diagnóstico de hidatidosis confirmado, señalando incluso el método utilizado para ello en el formulario de enfermedades de notificación obligatoria. Se podría presumir más bien que las cifras de los egresos hospitalarios podrían sobrevalorar los estimadores poblacionales de riesgo, considerando que en la realidad los pacientes que son hospitalizados varias veces a consecuencia de la hidatidosis debido a la ocurrencia de complicaciones que determinan más de un registro para el mismo paciente. Nótese además que no siempre estos pacientes tienen la confirmación diagnóstica con métodos de laboratorio.

A fin de estimar la magnitud de la subnotificación de esta enfermedad se debiera disponer de datos de prevalencia serológica, mediante la cuantificación de anticuerpos específicos en población aparentemente sana. De acuerdo a lo revisado hasta ahora, sólo los indicadores de riesgo relevantes para la toma de decisiones serían los obtenidos a partir del sistema de notificación obligatoria elaborado para estos fines y no los de egresos hospitalarios, el que también generan información útil para describir la enfermedad pero que han sido establecidos con otros fines. Sin embargo, debe destacarse que ambas fuentes de información son complementarias, con las fortalezas y debilidades ya comentadas.

La tasa de incidencia promedio para el período (2,2/100.000 hbtes) generada por el sistema de notificación es coincidente a lo referido por la $\mathrm{OPS}^{14}$, la que muestra que esta tasa se ha mantenido estable desde
1992, con valores entre 2 y 2,5 casos $/ 100.000$ hbtes $^{14}$. Sin embargo, la aparición anual de casos nuevos indicaría que ésta es una enfermedad cuyo agentes etiológico se encuentra presente en Chile, aun cuando su incidencia se ha mantenido estable en el período analizado. Las regiones de mayor incidencia fueron Aysén (38,2/100.000 hbtes), Magallanes (9,2/100.000 hbtes) y Coquimbo (5,0/100.000 hbtes). Morales y cols ${ }^{25}$, coinciden en que Aysén y Magallanes son las regiones más afectadas; sin embargo, con cifras muy superiores a las obtenidas en este estudio. Si bien la región de Coquimbo no pertenece a una zona del país considerada ganadera, excepto algunas zonas específicas rurales, un estudio de seroprevalencia en una zona de esta región estimó una tasa de 2,5/100.000 hbtes $^{21}$, cifra inferior a la tasa de egresos calculada para esa región (10,6/100.000 hbtes).

Destaca el alto número de casos notificados ocurridos en Bío-Bío y Los Lagos durante el período, concentrando el 23,1 y $13,2 \%$ del total de casos del período respectivamente, lo que permitiría plantear algunas inquietudes respecto a posibles mayores gastos en salud por concepto de hospitalizaciones, tratamientos y licencias médicas asociadas a la hidatidosis humana, las que debieran ser respondidas con estudios relacionados a los impactos de esta enfermedad, al menos en términos económicos.

En relación a las tasas obtenidas de los egresos hospitalarios se obtuvo una tasa promedio de 6,0 casos $/ 100.000$ hbtes, lo que difiere a la tasa de egreso indicada por Lorca y cols, de 9,7/100.000 hbtes ${ }^{21}$. Las regiones más comprometidas son Aysén con 43,8 por 100.000 hbtes, La Araucanía (28,1/100.000 hbtes) y Magallanes (16,4/100.000 hbts). La tendencia observada en el período al descenso de los egresos no permite deducir que exista una ocurrencia menor de casos enfermos y/o una menor subnotificación ya que como se mencionó anteriormente, los registros de éstos sólo dan cuenta de los casos hospitalizados y diagnósticos de hidatidosis que no siempre son confirmados.

Se destaca la gran diferencia observada entre la tasa de egreso y de incidencia en La Araucanía. La tasa de egreso de 28,1/100.000 hbtes calculada para el período supera en 7,6 veces la tasa de incidencia de 3,7/100.000 hbtes. Estos valores son menores a los reportados por Aliaga y Oberg $^{26}$, para el año 1998 (tasas de egresos entre 34,6 a $132,3 / 100.000$ hbtes y tasas de incidencia entre 13,4 y 18,0/100.000 hbtes. en diferentes comunas de la Región de Aysén). Las diferencias detectadas en este estudio debieran ser analizadas mediante un estudio más acabado de la situación en esta región.

\section{Conclusión}

Los resultados obtenidos muestran que la hidatidosis está presente en todo el territorio nacional, observándose 
que las tasas de incidencia del período estudiado de 2,2/100.000 hbtes han permanecido estables a pesar de los esfuerzos realizados. Esta enfermedad afecta principalmente a personas en plena edad productiva y sin diferencias significativas por sexo, por lo que sería de interés estimar los costos económicos relacionados con días de ausentismo laboral, tratamientos, hospitalizaciones, etc. Atención debiera ponerse también en personas con edades extremas.

A partir de egresos hospitalarios se observa una tendencia a la disminución en su presentación, pero como se trata de una zoonosis crónica, podría éstos reflejar infecciones de años previos. No todos los casos de hidatidosis ingresados al sistema de notificación obligatoria necesariamente son hospitalizados, por lo tanto, la subnotificación podría ser mejor cuantificada por estudios de seroprevalencia con representatividad nacional.

Las regiones más afectadas según la incidencia fueron Aysén, Magallanes y Coquimbo. Esta tendencia al ascenso hacia el sur del país está asociada a la mayor actividad ganadera y tenencia de perros. Se destaca la Región de La Araucanía por las diferencias observadas entre sus tasa de incidencia y de egresos. Deben plantearse nuevas hipótesis para abordar la situación particular de la hidatidosis en las regiones con mayores incidencias que tomen en cuenta condiciones específicas propias de cada región.

Se hace necesario destacar la pertinencia de reforzar con programas de capacitación a todos los participantes del sistema de notificación obligatoria de enfermedades y reforzar los sistemas de control y vigilancia epidemiológica. Especial énfasis se debiera poner en lo referido a las capacidades diagnósticas locales de todas las especies presentes de Echinococcus en Chile.

Agradecimientos. A Eugenia Guerra y Lucy Espinoza, Departamento de Estadísticas e Información en Salud, Ministerio de Salud, Chile. Junio de 2007.

\section{Resumen}

La hidatidosis humana en Chile se describe a partir de fuentes de información secundarias (bases de datos de enfermedades de notificación obligatoria y egresos hospitalarios) entre los años 2001 y 2005. Para valorar la magnitud de la infección en humanos se calcularon las tasas de incidencia y egresos hospitalarios en el período y según región geográfica. La incidencia promedio del período según notificaciones fue de 2,2 por 100.000 habitantes, con mayores tasas en las regiones de Coquimbo, La Araucanía y Magallanes. La tasa promedio del período según egresos fue de 6 por 100.000 habitantes siendo La Araucanía, Aysén y Magallanes las más afectadas. E1 58,8\% de casos notificados fueron por Echinococcus granulosus, mientras que $43,6 \%$ se registraron como equinococosis no especificada. El sistema notificación obligatoria da una estimación real de la magnitud de esta enfermedad, al reportar casos con confirmación diagnóstica. Las tasas obtenidas de los egresos sobreestiman la magnitud. Se recomienda concentrar los esfuerzos en el sistema de notificación y en potenciar estudios de seroprevalencia.

\section{Referencias}

1.- Vera G, Venturelli F, Ramírez J, Venturelli A. Hidatidosis humana. Cuad Cir 2003; 17: 88-94.

2.- Larrieu E, Belloto A, Arambulo III P, Tamayo H. Equinocococis quística: Epidemiología y Control en América del Sur. Parasitol Latinoam 2004; 59: 82-9.

3.- Butte J, Mege R, Caracci M, Báez S, Díaz A, Viñuela E, et al. Hidatidosis alveolar hepática. Rev Chil Cir 2004; 56 (6): 593-7.

4.- Organización de las Naciones Unidas para la Agricultura y la Alimentación (FAO). Estimación del impacto económico de la equinococosis quística en el cono sur (Argentina, Brasil, Chile y Uruguay) 2007.

5.- Muñoz P. Diagnóstico y tratamiento de la hidatidosis (comentario editorial). Rev Chil Infectol 2007; 24 (2): 153-4.

6.- Serra I. Hidatidosis alveolar, una enfermedad inexistente en Chile (carta al editor). Rev Chil Cir 2005; 57 (3): 278.
7.- Frider B, Larrieu E, Odrizola M. Long term outcome of asymptomatic liver hydatidosis. J Hepatol 1999; 30: 228-31.

8.- Larrieu E, Del Carpio M, Costa M, Yadon Z. Risks factors for hydatidosis in children of Rio Negro Province. A study of cases and control. Ann Trop Med Parasitol 2002; 96: 43-52.

9.- Alarcón C. Encuesta hospitalaria sobre hidatidosis humana en la Provincia de Valdivia, período 1992-1998. Tesis M.V. Universidad Austral de Chile. Valdivia Chile. 2000, 98 pp.

10.- Pérez J, Pérez A, Valenzuela M. Quistes hidatídicos primitivos de localización extraabdominal y extrapulmonar. Rev Chil Cir 1999; 51: 341-6.

11.- Manterola C, Vial M, Fonseca G, Carrasco R, Bustos M L, Muñoz N S, et al. Hidatidosis abdominal de localización extra hepática: características clínicas y evolución de una serie de casos. Rev Chil Cir 2002; 54 (2): 128-34.

12.- Pinto P. Hidatidosis hepática. Estudio de una serie de 534 casos. Rev Chil Cir 1991; 43: 184-7 13.- Manterola C, Molina E, Fernández O,
Garrido L, Ascencio L, Barroso M. Quistectomía subtotal. Una alternativa quirúrgica racional en el tratamiento de la hidatidosis hepática. Rev Chil Cir 1998; 50: 621-9.

14.- Organización Panamericana de la Salud (OPS). Informe del Proyecto Subregional Cono Sur de Control y Vigilancia de la Hidatidosis Argentina, Brasil, Chile y Uruguay ( $1^{\mathrm{a}}$ reunión). Uruguay; 2004.

15.- Larrieu E, Frider B, del Carpio M, Salvitti J, Mercapide C, Pereyra R, et al. Portadores asintomáticos de hidatidosis: epidemiología, diagnóstico y tratamiento. Rev Pan Salud Pública 2000; 8 (4): 250-6.

16.- Gatti A. Estandarización de una técnica de enzimoinmunoensayo para el diagnóstico de la hidatidosis humana. A Bioq Clin Latinoamer 1996; 30: 333-42.

17.- Franchi C, Di Vico B, Teggi A. Long-term evaluation of patients with hidatidosis treated with benzimidazole carbamates. Clin Infect Dis 1999; 29: 304-9. 
18.- Ministerio de Salud de Chile. Reglamento sobre notificación de enfermedades transmisibles de notificación obligatoria. Decreto 158. Ministerio de Salud 2004. URL disponible en http://epi.minsal.cl/epi/html/frames/frame8.htm (acccedido 24 abril de 2008).

19.- Ministerio de Salud de Chile. Boletín Declaración de enfermedades de Notificación Obligatoria. URL disponible en http://epi. minsal.cl/epi/html/frames/frame8.htm (accedido 24 abril de 2008).

20.- Ministerio de Salud de Chile. Norma técnica que establece uso de formulario "Informe Estadístico de Egreso Hospitalario" para la producción de información estadística sobre causas de egreso hospitalario y variables asociadas. Subsecretaría de Salud Pública,
Departamento de Asesoría Jurídica. 2007. URL disponible en www.minsal.cl/juridico/ DECRETO_127_07.doc (accedido 22 abril de 2008.

21.- Lorca M, Garrido M, Contreras M, Salinas P, Álvarez C, Ruiz M, et al. Seroprevalencia de hidatidosis humana en la región de Coquimbo. Chile. Parasitol Latinoam 2006; 61: 111-6.

22.- Organización Panamericana de la Salud (OPS) Clasificación Estadística Internacional de Enfermedades y Problemas relacionados con la Salud. Décima revisión. 1995.

23.- Ministerio de Salud de Chile. Proyecciones de población por grupos de edad y sexo, según Servicio de Salud y Comuna 2001- 2005. Departamento de Estadísticas e Información de Salud. URL disponible en: http://deis.minsal.cl/ deis/codigo/neuw/epobla.asp (accedido 24 de abril de 2008).

24.- Lorca M, Campanella C, Gavrilovics A, Lagos L, Florín C. Estimación de los costos totales y parciales de diagnóstico, tratamiento quirúrgico y seguimiento de la hidatidosis hepática en el Hospital San Juan de Dios, Santiago, Chile 1990-1999. Rev Chil Cir 2003; 55 (1): 60-4.

25.- Morales M, Luengo J, Vásquez J. Evolución de las tasas de morbilidad por hidatidosis en Chile desde 1989 a 1995. Avances en Ciencias Veterinarias 1998; 13 (2).

26.- Aliaga F, Oberg C. Epidemiología de la hidatidosis humana en la IX Región de la Araucanía, Chile.: 1991-1998. Bol Chil Parasitol 2000; 55 (3-4): 54-8. 